\section{Appropriate Screening for Leishmaniasis before \\ Immunosuppressive Treatments}

To the Editor: We read with great interest the article by Xynos et al. reporting 2 cases of leishmaniasis in patients treated with biologic drugs (1). Although we agree with most of the article, we are not totally convinced that serologic analysis alone could be used to screen for leishmaniasis before initiation of biologic or immunosuppressive treatments. Evidence indicates that serologic analysis can identify only symptomatic or asymptomatic cases with recent and still active infection $(2,3)$.

Leishmania spp. are pathogens that infect hematopoietic cells, where they establish chronic intracellular parasitism and survive for an infected person's lifetime. In leishmaniasisendemic countries, asymptomatic visceral leishmaniasis (VL) infections occur more frequently than clinically apparent VL cases. An estimated $10 \%-20 \%$ of persons with asymptomatic infections develop clinically overt VL (4). The leishmanin skin test (LST) (Montenegro test), an intradermal injection of a suspension of killed promastigotes, measures delayed hypersensitivity reactions and appears to be the only screening test capable of detecting asymptomatic leishmania infections.

A positive LST result is thought to indicate durable cell-mediated immunity after asymptomatic infection or clinical cure of VL. LST positivity may appear months to years postinfection, but once positivity appears, it persists in immunocompetent patients. A survey of VL in Ethiopia showed LST positivity in $32.2 \%$ of the population, but leishmania antibodies were found in only $4.1 \%(5)$.
Because different antigen preparations may affect test sensitivity, LST should use promastigotes of the Leishmania spp. present in an area. We believe that ideal screening for leishmaniasis should include LST along with serologic analysis. Unfortunately, little data exist on the use of antileishmania therapies for LST-positive or serologically positive patients. VL with unusual signs and symptoms may develop in immunocompromised patients with previous LST positivity after immunosuppressive treatments. Information about LST positivity is useful for calling attention to this potential risk for VL that may have unusual manifestations in these persons.

\section{Antonio Cascio and Chiara laria}

Author affiliations: University of Messina, Messina, Italy (A. Cascio); and Sapienza University of Rome, Rome, Italy (C. Iaria)

\section{DOI: 10.3201/eid1510.090881}

\section{References}

1. Xynos ID, Tektonidou MG, Pikazis D, Sipsas NV. Leishmaniasis, autoimmune rheumatic disease, and anti-tumor necrosis factor therapy, Europe. Emerg Infect Dis. 2009;15:956-9. DOI: 10.3201/ eid1506.090101

2. Barao SC, de Fonseca Camargo-Neves VL, Resende MR, da Silva LJ. Human asymptomatic infection in visceral leishmaniasis: a seroprevalence study in an urban area of low endemicity. Preliminary results. Am J Trop Med Hyg. 2007;77:1051-3.

3. Braz RF, Nascimento ET, Martins DR, Wilson ME, Pearson RD, Reed SG, et al. The sensitivity and specificity of Leishmania chagasi recombinant $\mathrm{K} 39$ antigen in the diagnosis of American visceral leishmaniasis and in differentiating active from subclinical infection. Am J Trop Med Hyg. 2002;67:344-8.

4. Badero R, Jones TC, Carvalho EM, Sampaio D, Reed SG, Barral A, et al. New perspectives on a subclinical form of visceral leishmaniasis. J Infect Dis. 1986;154:1003-11.

5. Hailu A, Berhe N, Sisay Z, Abraham I, Medhin G. Seroepidemiological and leishmanin skin test surveys of visceral leishmaniasis in south and southwest Ethiopia. Ethiop Med J. 1996;34:11-23.
Address for correspondence: Antonio Cascio, Unita' Operativa di Medicina Tropicale e Parassitologia, Policlinico "G. Martino," Via Consolare Valeria n. 1, 98125 Messina, Italy; email: acascio@unime.it

In Response: In their letter responding to our recently published article (1) Cascio and Iaria spark an important discussion on the usefulness of screening for Leishmania infection before administering biologic agents or other immunosuppressive treatments to patients having autoimmune rheumatic diseases and living in areas where Leishmania parasites are endemic (2). Although we agree in principle that early detection of asymptomatic Leishmania infection will decrease the incidence of the disease in immunosuppressed patients, current diagnostic tools may have a limited (or restricted) role in detecting Leishmania infection in this vulnerable patient population. Screening for leishmaniasis has been hampered by the lack of a standard test. Currently available serologic methods have variable sensitivities, specificities, and cross-reactivities, depending on the species being tested and the region where tests are performed. Many experts believe that serologic tests may complement other existing diagnostic tools, raising cost-efficiency concerns, especially in financially deprived countries (3).

A positive leishmanin skin test (LST) result indicates exposure to Leishmania spp. and is generally thought to reflect a durable cell-mediated immune response. No crossreaction occurs in patients with Chagas disease, but some cross-reactions are found in patients with glandular tuberculosis or lepromatous leprosy (4). Sustained positive responses have been documented for up to 20 years after exposure to the Leishmania parasite. Nevertheless, LST has 
limitations. In a longitudinal study of visceral leishmaniasis in Bangladesh, Bern et al. reported loss of LST sensitivity attributed to antigen-production issues, such as standardization and documentation of sensitivity, potency, and stability of leishmanin antigens (5). Also, prior treatment with immunosuppressive agents, which influence cell-mediated immunity, may decrease LST prognostic potency similarly to changes observed for the tuberculin skin test in similar settings $(6)$.

Variations in specificities and sensitivities limit the diagnostic potential of available diagnostic tools. The context of immunosuppression further contributes to the diagnostic complications and increases the need for additional research in leishmaniasis diagnostics.

\section{loannis D. Xynos, Maria G. Tektonidou, Dimitrios Pikazis, and Nikolaos V. Sipsas}

Author affiliations: National and Kapodistrian University of Athens, Athens, Greece (I.D. Xynos, D. Pikazis, N.V. Sipsas); and Euroclinic Hospital of Athens, Athens (M.G. Tektonidou).

\section{References}

1. Xynos ID, Tektonidou MG, Pikazis D, Sipsas NV. Leishmaniasis, autoimmune rheumatic disease, and anti-tumor necrosis factor therapy, Europe. Emerg Infect Dis. 2009;15:956-9. DOI: 10.3201/ eid1506.090101

2. Cascio A, Iaria C. Appropriate screening for leishmaniasis before immunosuppressive treatments. Emerg Infect Dis. 2009; $15: 1706$

3. Zijlstra EE, Nur Y, Desjeux P, Khalil EA, El-Hassan AM, Groen J. Diagnosing visceral leishmaniasis with the recombinant K39 strip test: experience from the Sudan. Trop Med Int Health. 2001;6:108-13. DOI: $10.1046 / \mathrm{j} .1365-3156.2001 .00680 . \mathrm{x}$

4. Singh S, Sivakumar R. Recent advances in the diagnosis of leishmaniasis. J Postgrad Med. 2003;49:55-60. DOI: 10.4103/00223859.927
5. Bern C, Amann J, Haque R, Chowdhury R, Ali M, Kurkjian KM, et al. Loss of leishmanin skin test antigen sensitivity and potency in a longitudinal study of visceral leishmaniasis in Bangladesh. Am J Trop Med Hyg. 2006;75:744-8.

6. Lalvani A, Millington KA. Screening for tuberculosis infection prior to initiation of anti-TNF therapy. Autoimmun Rev. 2008;8:147-52. DOI: $10.1016 /$ j. autrev.2008.07.011
Corresponding author: Nikolaos V. Sipsas, Pathophysiology Department, School of Medicine, National and Kapodistrian University of Athens, Mikras Asias 75, Athens, 11527, Greece; email: nsipsas@med.uoa.gr

\section{Red Snappers}

\section{Erin E. McConnell}

for a moment pretend

you are not

the infallible house staff,

but the latest admission-

hacking putrid sputum

from your soulful depths

or your festering chest,

depending on your mood.

slapped with a mask,

you are secured in secluded rooms;

a paucity of guests,

but for the parade of absurd birds-

plastered in Haz-mat

lemon-yellow gowns,

and peach-colored beaks.

your meager dried-up sleep

is aborted by

bloodhungry fowl

covetous of mucus

you no longer produce.

your meals grow cold

waiting for you

in the anteroom of

your negative pressure purgatory.

Dr McConnell is a fourth-year resident in the Internal Medicine Pediatric Residency Program at Wayne State University, Dayton, Ohio, USA.

Author affiliation: Wright State University, Dayton, Ohio, USA

DOI: $10.3201 /$ eid1510.090221

Address for correspondence: Erin E. McConnell, Wright State University, Internal Medicine/ Pediatrics, 1 Elizabeth Place, NWG Suite 500, Dayton, OH 45408, USA; email: erin. mcconnell@wright.edu 